NISSUNA UMANA INVESTIGAZIONE SI PUO DIMANDARE VERA SCIENZIA S'ESSA NON PASSA PER LE MATEMATICHE DIMOSTRAZIONI LEONARDO DA VINCI

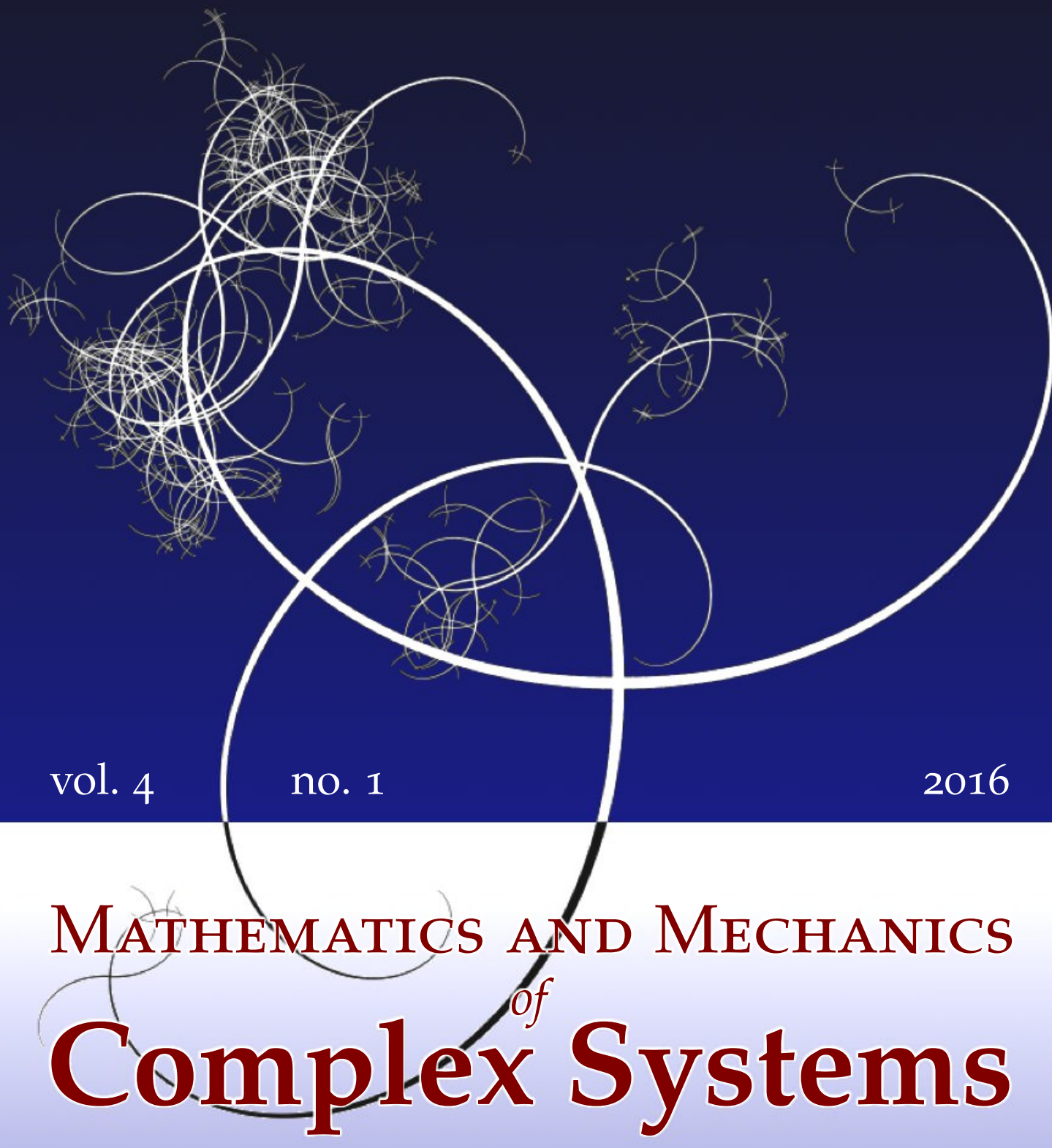

David J. Steigmann And Ashutosh Agrawal

ELECTROMECHANICS OF POLARIZED LIPID BILAYERS 


\title{
ELECTROMECHANICS OF POLARIZED LIPID BILAYERS
}

\author{
David J. Steigmann AND Ashutosh AgRawal
}

\begin{abstract}
A model for the electromechanics of lipid bilayers, accounting for flexoelectricity, is obtained as the thin-film limit of the continuum electrodynamics of nematic liquid crystals. A priori restrictions on the polarization field consistent with minimum energy considerations effectively decouple the leading-order membrane problem from the computation of the self field, yielding a substantial simplification vis a vis the three-dimensional theory. Examples illustrate the strong interplay between the electric field and membrane geometry.
\end{abstract}

\section{Introduction}

The idea that lipid bilayers can be regarded as thin liquid crystal films apparently originated in the work of Helfrich [1973]. This point of view gave rise to an associated body of work that has been thoroughly documented in [Ou-Yang et al. 1999]. The liquid-crystal framework provides a clear conceptual foundation for extensions of the basic purely mechanical theory to coupled-field problems. In the present work, we use this foundation to develop an electromechanical theory of lipid bilayers. This framework may be used to gain physical insight into various phenomena. For example, lipid vesicles have been shown to deform in the presence of applied electric fields [Winterhalter and Helfrich 1988; Kummrow and Helfrich 1991; Dimova et al. 2007; 2009; Vlahovska 2010]. The creation of nanopores in lipid membranes by external electric fields is a standard technique - known as electroporation - to deliver genes into cells [Neumann et al. 1982; Aihara and Miyazaki 1998; Weaver 2000] and in some cancer treatments [Davalos et al. 2005; Rubinsky et al. 2007]. The role of coupled electromechanical interactions is well recognized in the context of cochlear outer hair cells [Brownell et al. 1985; Raphael et al. 2000; Harland et al. 2015]. Electromechanical interactions also play a fundamental role in electrically active cells such as neurons. Further, experimental studies have revealed that voltage-gated ion channels exhibit sensitivity to both electrostatic and mechanical forces [Schmidt et al. 2012].

\section{Communicated by Francesco dell'Isola.}

MSC2010: 74P10.

Keywords: electromechanics, lipid membranes. 
Here we adapt the three-dimensional liquid crystal theory advanced in [de Gennes and Prost 1992; Ericksen 1961; 1962; 1976; Virga 1994] to derive a two-dimensional model for the response of electrically polarized lipid bilayers to applied electric fields generated by a remote source. In this respect our approach differs substantially from recent efforts directed at modeling electromechanical interactions in lipid membranes [Gao et al. 2008; Mohammadi et al. 2014]. For definiteness and for the sake of simplicity, we base our model on the general theory for nematics [Virga 1994], incorporating modifications associated with the so-called flexoelectric effect [Meyer 1969; de Gennes and Prost 1992].

In Section 2 we summarize those aspects of the basic three-dimensional theory that are required for our purpose. This is based on an extension to liquid-crystal theory of an expression for the potential energy of a polarized material, subject to a remotely generated applied electric field regarded as an assigned function of position in the ambient space [Toupin 1956; Truesdell and Toupin 1960; Bustamante et al. 2009; Dorfmann and Ogden 2014]. Insofar as electrical interactions are concerned, we confine attention in this preliminary work to the effects of polarization and assume free charges to be absent. The relevant three-dimensional energy is used, in Section 3, to derive the leading-order-in-thickness expression for the energy of the two-dimensional model. The operative equilibrium equations and edge conditions are derived from this via a variational procedure in Sections 4 and 5 , respectively, and the theory is illustrated through numerical solution of several examples involving axisymmetry in Section 6. We freely use the standard notation of the classical differential geometry of surfaces. The text by Sokolnikoff [1964] is recommended for mechanicians seeking a comprehensive treatment.

\section{Energetics of three-dimensional liquid crystals in the presence of a stationary applied field}

Numerous variational formulations of electromechanical interactions in deformable media are available in the literature. These have been extensively examined and correlated in [Bustamante et al. 2009; Dorfmann and Ogden 2014], to which the interested reader is referred for fuller expositions. There it is shown that Maxwell's equations and the equilibrium equations for a polarized medium in the presence of an applied electric field that is fixed in space, in the absence of applied loads or free electric charges, render stationary the energy functional

$$
\mathcal{E}=\int_{R}\left(U-\frac{1}{2} \boldsymbol{e}_{s} \cdot \boldsymbol{p}-\boldsymbol{e}_{a} \cdot \boldsymbol{p}\right) d v,
$$

where $R$ is the volume currently occupied by the material in three-space, $U$ is the relevant energy density, $\boldsymbol{p}$ is the polarization per unit volume, $\boldsymbol{e}_{s}$ is the electric self field generated by the polarized material, and $\boldsymbol{e}_{a}$ is the applied electric field, 
assumed to be assigned as a smooth function in all of three-space, including $R$. The net electric field is

$$
\boldsymbol{e}=\boldsymbol{e}_{s}+\boldsymbol{e}_{a} .
$$

Further, the applied field is a fixed function of position $\boldsymbol{y}$ in the enveloping threespace. Its variational derivative, associated with a fixed material point (a fixed lipid molecule in the present context), is thus purely convective; i.e.,

$$
\dot{\boldsymbol{e}}_{a}=\left(\operatorname{grad} \boldsymbol{e}_{a}\right) \dot{\boldsymbol{y}}
$$

where grad is the (spatial) gradient with respect to $\boldsymbol{y}$; and, here and henceforth, superposed dots are used to denote variational - or Gateaux - derivatives. The field $\boldsymbol{e}_{a}$ is curl-free; accordingly, its gradient is symmetric: $\operatorname{grad} \boldsymbol{e}_{a}=\left(\operatorname{grad} \boldsymbol{e}_{a}\right)^{t}$. The self field is also curl-free; it is obtained from

$$
\boldsymbol{e}_{s}=-\operatorname{grad} V_{s},
$$

where the self field potential $V_{s}$ is given by [Kovetz 2000]

$$
4 \pi \varepsilon_{0} V_{S}(\boldsymbol{y})=\int_{\partial R} \frac{\boldsymbol{p}^{\prime} \cdot \boldsymbol{n}^{\prime}}{\left|\boldsymbol{y}-\boldsymbol{y}^{\prime}\right|} d a-\int_{R} \frac{\operatorname{div}^{\prime} \boldsymbol{p}^{\prime}}{\left|\boldsymbol{y}-\boldsymbol{y}^{\prime}\right|} d v,
$$

in which $\varepsilon_{0}$ is the free-space permittivity; $\boldsymbol{n}^{\prime}$ is the exterior unit normal to $\partial R$, expressed as a function of the integration variable $\boldsymbol{y}^{\prime} ; \boldsymbol{p}^{\prime}$ is likewise the polarization in terms of $\boldsymbol{y}^{\prime}$; and $\operatorname{div}^{\prime}$ is the divergence with respect to $\boldsymbol{y}^{\prime}$. This is defined by $\operatorname{div} \boldsymbol{p}=\operatorname{tr}(\operatorname{grad} \boldsymbol{p})$, where $\operatorname{tr}(\cdot)$ is the trace.

The energy density $U$ is a function of the polarization and appropriate deformation variables. In the conventional theory of electroelasticity the relevant deformation variable is the deformation gradient, the gradient of $\boldsymbol{y}=\chi(\boldsymbol{x})$ with respect to position $\boldsymbol{x}$ in some fixed reference configuration. Here $\chi(\cdot)$ is a field describing the deformation of material points. In the present application to liquid crystals, the relevant variables are a director field $\boldsymbol{d}(\boldsymbol{y})$ - describing the orientation of the liquid crystal molecules — and its spatial gradient

$$
\boldsymbol{D}=\operatorname{grad} \boldsymbol{d} .
$$

We follow the conventional theory and impose $|\boldsymbol{d}(\boldsymbol{y})|=1$.

The electric field is given in terms of the polarization by the partial derivative [Toupin 1956; Bustamante et al. 2009]

$$
e=U_{p}(d, D, p) .
$$

In applications $U$ is typically assumed to be a quadratic function of $\boldsymbol{D}$. This reflects the notion that the length scale for spatial variations of the director is typically much larger than the local length scale: the molecular length; the dimensionless 
gradient is then sufficiently small to justify the termination of the Taylor expansion of $U(\boldsymbol{d}, \cdot, \boldsymbol{p})$ at second order. Thus,

$$
U=l(\boldsymbol{d}, \boldsymbol{p})+\boldsymbol{L}(\boldsymbol{d}, \boldsymbol{p}) \cdot \boldsymbol{D}+\frac{1}{2} \boldsymbol{D} \cdot \mathcal{L}(\boldsymbol{d}, \boldsymbol{p})[\boldsymbol{D}],
$$

in which $l, \boldsymbol{L}$ and $\mathcal{L}$ are scalar, second-order tensor and fourth-order tensor valued functions, respectively, with $\mathcal{L}=\mathcal{L}^{t}$.

Guided by [Virga 1994], we adopt the specific forms

$$
l(\boldsymbol{d}, \boldsymbol{p})=\frac{1}{2} \chi_{\perp}|\boldsymbol{p}|^{2}+\frac{1}{2} \chi_{a}(\boldsymbol{p} \cdot \boldsymbol{d})^{2},
$$

where $\chi_{\perp}$ and $\chi_{a}$ are the anisotropic dielectric constants, and

$$
\begin{aligned}
\boldsymbol{D} & \cdot \mathcal{L}(\boldsymbol{d}, \boldsymbol{p})[\boldsymbol{D}] \\
& =k_{1}(\operatorname{div} \boldsymbol{d})^{2}+k_{2}(\boldsymbol{d} \cdot \operatorname{curl} \boldsymbol{d})^{2}+k_{3}|\boldsymbol{D} \boldsymbol{d}|^{2}+\left(k_{2}+k_{4}\right)\left[\operatorname{tr}\left(\boldsymbol{D}^{2}\right)-(\operatorname{div} \boldsymbol{d})^{2}\right],
\end{aligned}
$$

in which the latter is independent of $\boldsymbol{p}$, and $k_{1}-k_{4}$ are constants with $2 k_{1} \geq k_{2}+k_{4}$, $k_{2} \geq\left|k_{4}\right|$ and $k_{3} \geq 0$, in accordance with the presumed positive-definiteness of $\mathcal{L}$ [Virga 1994]. The second expression is the Frank energy for nematic liquid crystals.

To model the flexoelectric effect, we adopt Meyer's proposal [1969] in a form similar to that used by Ou-Yang et al. [1999]. Thus,

$$
\boldsymbol{L}(\boldsymbol{d}, \boldsymbol{p}) \cdot \boldsymbol{D}=-\boldsymbol{p} \cdot \boldsymbol{f}(\boldsymbol{d}, \boldsymbol{D}), \quad \text { with } \boldsymbol{f}(\boldsymbol{d}, \boldsymbol{D})=c_{1}(\operatorname{div} \boldsymbol{d}) \boldsymbol{d}+c_{2} \operatorname{curl} \boldsymbol{d} \times \boldsymbol{d},
$$

where $c_{1}$ and $c_{2}$ are the flexoelectric constants. The relationship (7) then furnishes an expression for the electric field

$$
\boldsymbol{e}=\chi_{\perp} \boldsymbol{p}+\chi_{a}(\boldsymbol{p} \cdot \boldsymbol{d}) \boldsymbol{d}-\boldsymbol{f}(\boldsymbol{d}, \boldsymbol{D}) .
$$

This coincides, in the specialization $\chi_{a}=\chi_{\perp}$, with Equation (2.153) in [Ou-Yang et al. 1999] in the case when the electric field vanishes.

In the absence of polarization (8) reduces precisely to the conventional liquidcrystal energy. The stationarity of this energy is equivalent, under appropriate regularity conditions, to the well-known equilibrium equations and natural boundary conditions of liquid-crystal theory [de Gennes and Prost 1992; Ericksen 1976; Virga 1994; Steigmann 2013].

Our objective in the present work is to derive the leading-order small thickness limit of the energy (1). This limit is taken to be the energy of a polarized lipid membrane. Stationarity conditions for the limit energy are then identified with the equilibrium equations of a polarized lipid membrane in the presence of an applied field generated by a remote source.

We have in mind a lipid bilayer constituting a membrane structure in a typical animal cell. Because such a membrane is only one or two molecules across, its thickness is on the order of the local length scale embodied in the constitutive 
response of the liquid crystal. Accordingly, this is the only relevant length scale in the dimension reduction procedure. In contrast, in recent work on thin films of nematic elastomers [Cesana et al. 2015], the local length scale is always much smaller than the values of film thickness deemed to be relevant. The local scale therefore vanishes with thickness, leading to a reduced theory in which the director gradient ultimately plays no role. Of course it may be argued that reliance on threedimensional liquid crystal theory to effect a dimension reduction procedure may not be justified if the thickness is comparable to molecular dimensions. In this instance our procedure nevertheless furnishes guidance for the construction of a direct two-dimensional field theory.

\section{Liquid crystal films}

In the purely mechanical theory of thin liquid crystal films the leading-order strain energy density $W$ is associated with the limit [Steigmann 2013]

$$
\lim _{t \rightarrow 0} t^{-1} \int_{R} U d v=\int_{\omega} W d a,
$$

where $\omega$ is the interior midsurface of the film, $t$ is the (uniform) thickness of the film, and

$$
W=U_{\mid \omega}
$$

is the leading-order energy density on $\omega$. This follows by using the volume measure $d v=\mu d \varsigma d a$ [Naghdi 1972], where $\varsigma$ is a linear coordinate in the direction of the unit surface normal $\boldsymbol{n}$, regarded as the restriction of $\boldsymbol{d}$ to $\omega$, and $\mu=1-2 \varsigma H+\varsigma^{2} K$, where $H$ and $K$ respectively are the mean and Gaussian curvatures of $\omega$. In effect, then, we suppress misalignment of the lipid molecules with the surface normal the so-called lipid tilt — as in the classical Canham-Helfrich theory. This is appropriate if the surface density of the lipids is sufficiently high. Generalizations to accommodate tilt are described in [Steigmann 2013]. We have [Steigmann 2013]

$$
\boldsymbol{n}=\boldsymbol{d}_{\mid \omega} \quad \text { and } \quad \boldsymbol{D}_{\mid \omega}=\nabla \boldsymbol{n}+\boldsymbol{\eta} \otimes \boldsymbol{n},
$$

where $\nabla(\cdot)$ is the (two-dimensional) surface gradient on $\omega$ and $\eta$ is the restriction to $\omega$ of the derivative of $\boldsymbol{d}$ in the direction of $\boldsymbol{d}$. Accordingly,

$$
\boldsymbol{n} \cdot \boldsymbol{\eta}=0 \quad \text { on } \omega .
$$

The extension to the case of polarizable films in the presence of an applied field is immediate. Thus,

with

$$
\lim _{t \rightarrow 0} t^{-1} \mathcal{E}=E,
$$

$$
E=\int_{\omega} W d a
$$


where $W$ is now given by

$$
W=\left(U-\frac{1}{2} \boldsymbol{e}_{s} \cdot \boldsymbol{p}-\boldsymbol{e}_{a} \cdot \boldsymbol{p}\right)_{\mid \omega} .
$$

Remark 1. Quantum mechanical considerations and molecular dynamics simulations in respect of polarized lipid membranes [Seelig 1978; Frischleder and Peinel 1982; Warshaviak et al. 2011] indicate that the polarization vector is essentially tangential to the film surface. In this case an estimate based on (5) - derived in the mathematically identical context of magnetostatics [Barham et al. 2012] indicates that the magnitude $\left|\boldsymbol{e}_{s}\right|$ of the self field is of order $O(t \ln t)$; for small $t$ this is negligible compared to unity. It follows that the leading-order energy, i.e., the limit of $\mathcal{E} / t$ as $t \rightarrow 0$, is given by (18) but with $W$ simplified to

$$
W=U_{\mid \omega}-\boldsymbol{e}_{a}(\boldsymbol{r}) \cdot \pi,
$$

where $\boldsymbol{\pi}=\boldsymbol{p}_{\mid \omega}, \boldsymbol{r}=\boldsymbol{y}_{\mid \omega}$ is the position field on $\omega$, and

$$
\boldsymbol{n} \cdot \boldsymbol{\pi}=0 \quad \text { on } \omega .
$$

Remark 2. The estimate on the self field effectively decouples its computation from the problem of rendering $E$ stationary, implying, in particular, that it may be evaluated a posteriori. This feature affords a major simplification of the theory for thin films vis a vis that for bulk continua. Further, in the analogous magnetostatic setting, the condition (21), with polarization replaced by magnetization, is known to furnish energetically optimal states of magnetization in thin films [Gioia and James 1997]. Thus our approach via dimension reduction provides justification for the suppression of the self field, in the leading order two-dimensional model, under conditions in which the polarization field is tangential to the membrane. In contrast, in [Gao et al. 2008; Mohammadi et al. 2014] no analysis is offered to justify the suppression of the self field.

The self field at points in space remote from the membrane may be evaluated a posteriori by applying the divergence theorem to (5), for points $y$ not in the closure of $R$. This furnishes

$$
4 \pi \varepsilon_{0} V_{s}(\boldsymbol{y})=\int_{R} \boldsymbol{p}^{\prime} \cdot \frac{\boldsymbol{y}-\boldsymbol{y}^{\prime}}{\left|\boldsymbol{y}-\boldsymbol{y}^{\prime}\right|^{3}} d v=t\left[\int_{\omega} \boldsymbol{\pi} \cdot \frac{\boldsymbol{y}-\boldsymbol{r}}{|\boldsymbol{y}-\boldsymbol{r}|^{3}} d a+o(t) / t\right],
$$

in which $\boldsymbol{r}$ is the membrane position field. The self field then follows by computing the gradient with respect to $\boldsymbol{y}$ (cf. (4)), yielding

$$
4 \pi \varepsilon_{0} \lim _{t \rightarrow 0}\left(t^{-1} \boldsymbol{e}_{s}\right)=\int_{\omega} \boldsymbol{G} \boldsymbol{\pi} d a,
$$

where

$$
\boldsymbol{G}=\frac{3}{|\boldsymbol{y}-\boldsymbol{r}|^{5}}(\boldsymbol{y}-\boldsymbol{r}) \otimes(\boldsymbol{y}-\boldsymbol{r})-\frac{1}{|\boldsymbol{y}-\boldsymbol{r}|^{3}} \boldsymbol{I}
$$


The leading-order self field in space is thus delivered by a quadrature over $\omega$ after membrane shape has been determined.

In the expression (20) we have

$$
U_{\mid \omega}=U(\boldsymbol{n},-\boldsymbol{b}+\boldsymbol{\eta} \otimes \boldsymbol{n}, \boldsymbol{\pi}),
$$

where

$$
\boldsymbol{b}=-\nabla \boldsymbol{n}
$$

is the (symmetric) curvature tensor on $\omega$; at any particular point of $\omega$ this maps the tangent plane $T_{\omega}$ to itself.

The explicit form used here follows from (8)-(11). For example, the restriction to $\omega$ of the function $\boldsymbol{f}(\boldsymbol{d}, \boldsymbol{D})$ in (11) is given by

$$
\boldsymbol{f}_{\mid \omega}=c_{1}(\boldsymbol{n} \cdot \boldsymbol{\eta}-2 H) \boldsymbol{n}+c_{2} \boldsymbol{\eta},
$$

where

$$
H=\frac{1}{2} \operatorname{tr} \boldsymbol{b}
$$

is the mean curvature of $\omega$. This expression may be simplified by imposing (16) but we refrain from doing so for reasons to be discussed later. Here use has been made of (15) and the formula curl $\boldsymbol{d} \times \boldsymbol{d}=\boldsymbol{D} \boldsymbol{d}$, which follows from the fact that $\boldsymbol{d}$ is a field of unit vectors [Virga 1994].

To reduce (10) we first introduce a coordinate parametrization $\boldsymbol{r}\left(\theta^{\alpha}\right)$ of $\omega$. This induces the natural tangent basis $\boldsymbol{a}_{\alpha}=\boldsymbol{r}_{, \alpha} \in T_{\omega}$ and associated dual basis $\boldsymbol{a}^{\alpha} \in T_{\omega}$, where $(\cdot)_{, \alpha}=\partial(\cdot) / \partial \theta^{\alpha}$. Then, the restriction of curl $\boldsymbol{d}$ to $\omega$ is [Steigmann 2013]

$$
(\operatorname{curl} \boldsymbol{d})_{\mid \omega}=\boldsymbol{a}^{\alpha} \times \boldsymbol{n}_{, \alpha}+\boldsymbol{n} \times \boldsymbol{\eta},
$$

where $\boldsymbol{a}^{\alpha} \times \boldsymbol{n}_{, \alpha}=-b_{\alpha \beta} \boldsymbol{a}^{\alpha} \times \boldsymbol{a}^{\beta}$, with $b_{\alpha \beta}=\boldsymbol{a}_{\alpha} \cdot \boldsymbol{b} \boldsymbol{a}_{\beta}$, vanishes by virtue of the symmetry of $\boldsymbol{b}$; accordingly, $(\boldsymbol{d} \cdot \operatorname{curl} \boldsymbol{d})_{\mid \omega}=0$.

Using (26) with $\boldsymbol{b} \boldsymbol{n}=\mathbf{0}$ we also derive

$$
\operatorname{tr}\left(\boldsymbol{D}^{2}\right)_{\mid \omega}=\operatorname{tr}\left(\boldsymbol{b}^{2}\right)+(\boldsymbol{n} \cdot \boldsymbol{\eta})^{2} .
$$

Applying the Cayley-Hamilton formula

$$
\boldsymbol{b}^{2}=2 H \boldsymbol{b}+K \mathbf{1}
$$

where

$$
K=\operatorname{det} \boldsymbol{b}
$$

is the Gaussian curvature of $\omega$ and $\mathbf{1}$ is the identity transformation on $T_{\omega}$, we then obtain

$$
\left[\operatorname{tr}\left(\boldsymbol{D}^{2}\right)-(\operatorname{div} \boldsymbol{d})^{2}\right]_{\mid \omega}=2 H \boldsymbol{n} \cdot \boldsymbol{\eta}-2 K,
$$

which again may be simplified by imposing (16). 
Remark 3. It is well known [Virga 1994] that the combination $\operatorname{tr}\left(\boldsymbol{D}^{2}\right)-(\operatorname{div} \boldsymbol{d})^{2}$ is a null Lagrangian in the three-dimensional theory. It is also well known that the Gaussian curvature is a null Lagrangian in the surface theory; in particular, the total curvature of a closed surface is fixed by its genus and thus contributes only a disposable constant to the energy if the latter is invariant, as we assume in the present work. Accordingly, (16) implies that the same combination of terms also furnishes a null Lagrangian in the two-dimensional theory.

Altogether, the surface energy reduces to (cf. (8))

$$
\begin{aligned}
U_{\mid \omega}=\frac{1}{2} \chi_{\perp}|\boldsymbol{\pi}|^{2}+ & \frac{1}{2} \chi_{a}(\boldsymbol{\pi} \cdot \boldsymbol{n})^{2}-c_{1}(\boldsymbol{n} \cdot \boldsymbol{\eta}-2 H) \boldsymbol{n} \cdot \boldsymbol{\pi}-c_{2} \boldsymbol{\eta} \cdot \boldsymbol{\pi} \\
& +\frac{1}{2} k_{1}(\boldsymbol{n} \cdot \boldsymbol{\eta}-2 H)^{2}+\frac{1}{2} k_{3}|\boldsymbol{\eta}|^{2}+\left(k_{2}+k_{4}\right)(H \boldsymbol{n} \cdot \boldsymbol{\eta}-K),
\end{aligned}
$$

yielding the net energy density in the form

$$
W=k H^{2}+\bar{k} K+\frac{1}{2} k_{3}|\boldsymbol{\eta}|^{2}+\frac{1}{2} \chi_{\perp}|\boldsymbol{\pi}|^{2}-c_{2} \boldsymbol{\eta} \cdot \boldsymbol{\pi}+\tilde{\varphi} \boldsymbol{n} \cdot \boldsymbol{\pi}+\tilde{\psi} \boldsymbol{n} \cdot \boldsymbol{\eta}-\boldsymbol{e}_{a}(\boldsymbol{r}) \cdot \boldsymbol{\pi},
$$

where

$$
k=2 k_{1}, \quad \bar{k}=-\left(k_{2}+k_{4}\right)
$$

and $\tilde{\varphi}, \tilde{\psi}$ are certain scalars which will prove to be irrelevant. Accordingly, $W$ may be regarded as a function of the list

$$
\{H, K, \boldsymbol{r}, \boldsymbol{n}, \boldsymbol{\eta}, \boldsymbol{\pi}\},
$$

subject to the constraints (16) and (21), in which it is understood that $H, K$ and $\boldsymbol{n}$ are determined by the parametrization $\boldsymbol{r}\left(\theta^{\alpha}\right)$. Henceforth we require the doublet $\left\{\theta^{\alpha}\right\}$ to maintain a fixed correspondence with a material point; i.e., a lipid molecule. Thus the coordinates are convected with the lipids in the course of any configurational variation.

\section{Variational problem and equilibrium equations}

It is convenient to adopt an extended variational formulation in which the constraints are relaxed. In this formulation we do not impose (16) or (21), but instead consider the auxiliary energy

$$
E^{*}=\int_{\Omega}[J W+\lambda(J-1)+\bar{\varphi} \boldsymbol{n} \cdot \boldsymbol{\pi}+\bar{\psi} \boldsymbol{n} \cdot \boldsymbol{\eta}] d A,
$$

where $W$ is given by (35), with $\boldsymbol{r}, \boldsymbol{\eta}$ and $\boldsymbol{\pi}$ regarded as independent fields, and where $\bar{\varphi}$ and $\bar{\psi}$ are Lagrange-multiplier fields associated with the constraints (16) and (21). Here $\Omega$ is the preimage of $\omega$ in a fixed reference placement, with $d a=J d A$. In terms of the convected-coordinate surface parametrization we have $J=\sqrt{a / A}$, where $a=\operatorname{det}\left(a_{\alpha \beta}\right), a_{\alpha \beta}=\boldsymbol{a}_{\alpha} \cdot \boldsymbol{a}_{\beta}$ is the surface metric, and $A$ is the value of $a$ on $\Omega$. Further, $\lambda$ is a Lagrange-multiplier field associated with the constraint that 
the map from any configuration to another preserves local surface area; and, hence, that $J=1$. This restriction is appropriate in the absence of lipid distension, as in the classical Canham-Helfrich theory; the bulk incompressibility of the liquid crystal then implies that area is preserved locally [Steigmann 2013]. Generalizations to account for distension in a purely mechanical setting are discussed in [Steigmann 2013; Kim and Steigmann 2015].

We observe that $\tilde{\varphi}$ and $\tilde{\psi}$ in (35) may be absorbed into the Lagrange multipliers and conclude that no generality is lost if (35) is replaced by

$$
W=U-e_{a}(\boldsymbol{r}) \cdot \pi
$$

in (38), where $U$ is now given by

$$
U=k H^{2}+\bar{k} K+\frac{1}{2} k_{3}|\eta|^{2}+\frac{1}{2} \chi_{\perp}|\boldsymbol{\pi}|^{2}-c_{2} \boldsymbol{\eta} \cdot \boldsymbol{\pi} .
$$

We note that the quadratic form involving $\boldsymbol{\eta}$ and $\boldsymbol{\pi}$ is positive definite if and only if $k_{3}>0, \chi_{\perp}>0$ and $c_{2}^{2}<k_{3} \chi_{\perp}$.

The expression (38) reduces to the actual energy when the constraints (16) and (21) are operative, and is well defined when they are not; it therefore furnishes an extension of the actual energy to arbitrary (unconstrained) states. Stationarity with respect to the multipliers simply returns the constraints as the relevant Euler-Lagrange equations. Moreover, stationarity of $E^{*}$ with respect to arbitrary variations implies stationarity with respect to constrained variations in particular, and hence stationarity of the actual energy $E$. We use this observation to derive equilibrium equations for the actual constrained system. We note that while the replacement of $E$ by $E^{*}$ is permissible for the purpose of extracting stationarity (i.e., equilibrium) conditions, it may not be used to study energy minimizers. This is a consequence of the fact that $\inf E^{*} \leq \inf E$, this following trivially from constraint relaxation.

The variational derivative of the extended energy, modulo the variations of the Lagrange multipliers, is

$$
\dot{E}^{*}=\int_{\omega}[\dot{W}+(W+\lambda) \dot{J} / J+\varphi(\dot{\boldsymbol{n}} \cdot \boldsymbol{\pi}+\boldsymbol{n} \cdot \dot{\boldsymbol{\pi}})+\psi(\dot{\boldsymbol{n}} \cdot \boldsymbol{\eta}+\boldsymbol{n} \cdot \dot{\boldsymbol{\eta}})] d a,
$$

where $\varphi=\bar{\varphi} / J$ and $\psi=\bar{\psi} / J$, and it is understood, having suppressed the variations of the multipliers, that all terms in this expression are to be evaluated, post facto, at states satisfying the constraints (16) and (21). In the presence of a net lateral pressure $p$ in the direction of the surface normal $\boldsymbol{n}$, the virtual-work statement is given by Agrawal and Steigmann [2009] as

$$
\dot{E}^{*}=\int_{\omega} p \boldsymbol{n} \cdot \dot{\boldsymbol{r}} d a+\int_{\partial \omega} \chi d s
$$


where $\chi$ is the density of edge power, the form of which will be made explicit below. We remark that because of the definition (14), the energy in this expression is actually the energy divided by the thickness $t$; the dimensions of $p$ and $\chi$ are affected accordingly. Thus, for example, the actual pressure is $t p$, and with $p=O(1)$ this is of order $t$.

We consider the consequences of (42) with respect to variations of each variable in turn. The simplest are those associated with the variations $\dot{\boldsymbol{\pi}}$ and $\dot{\eta}$. They are given respectively by

$$
\boldsymbol{e}_{a}=U_{\pi}+\varphi \boldsymbol{n}
$$

and

$$
U_{\eta}+\psi \boldsymbol{n}=\mathbf{0},
$$

(cf. (7) and (15)), with

$$
U_{\pi}=\chi_{\perp} \pi-c_{2} \eta \text { and } \quad U_{\eta}=k_{3} \boldsymbol{\eta}-c_{2} \pi .
$$

Accordingly, with the constraints (16) and (21) in effect it follows that

$$
\psi=0 \quad \text { and } \quad \boldsymbol{\eta}=\left(c_{2} / k_{3}\right) \pi
$$

and that

$$
\varphi=\boldsymbol{n} \cdot \boldsymbol{e}_{a} \quad \text { and } \quad U_{\pi}=\mathbb{P} \boldsymbol{e}_{a},
$$

where $\mathbb{P}=\boldsymbol{I}-\boldsymbol{n} \otimes \boldsymbol{n}$ is the projection onto $T_{\omega}$, with $\boldsymbol{I}$ the identity for 3 -space. We note that $\mathbb{P}=\mathbf{1}$, the identity on $T_{\omega}$. Then, from $(45)_{1}$,

$$
D \pi=\mathbb{P} \boldsymbol{e}_{a}(\boldsymbol{r}), \quad \text { where } D=\chi_{\perp}-c_{2}^{2} / k_{3},
$$

which furnishes the polarization uniquely in terms of the surface parametrization, provided that $D \neq 0$ and the applied field is assigned as a function in space. When $\chi_{\perp}>0$, the sign of $D$ is controlled by the strength of the flexoelectric effect; thus $D$ is positive or negative according as $\left|c_{2}\right|$ is small or large, respectively. These alternatives correspond to the relevant quadratic form in the energy being positive definite or indefinite, respectively.

These results imply that the equilibrium value of the energy (40) may be regarded as a function of $H, K$ and $\pi$; on combining (46) and (48), the explicit expression is found to be

$$
U=k H^{2}+\bar{k} K+\frac{1}{2} D|\pi|^{2} .
$$

Necessary conditions for minimum energy states in the absence of polarization, derived in [Agrawal and Steigmann 2008], require $k>0$ but do not impose any restriction on $\bar{k}$. 
With the foregoing in effect, (41) and (42) furnish the residual virtual-work statement

$$
\int_{\omega}[\dot{W}+(W+\lambda) \dot{J} / J+\varphi \boldsymbol{\pi} \cdot \dot{\boldsymbol{n}}] d a=\int_{\omega} p \boldsymbol{n} \cdot \dot{\boldsymbol{r}} d a+\int_{\partial \omega} \chi d s,
$$

in which all variations are induced by the virtual velocity

$$
\boldsymbol{u}=\dot{\boldsymbol{r}}
$$

with $\dot{\boldsymbol{\pi}}=\mathbf{0}$. In particular [Steigmann 2013],

$$
\dot{J} / J=\boldsymbol{a}^{\alpha} \cdot \boldsymbol{u}_{, \alpha}
$$

and

$$
\dot{\boldsymbol{n}}=\varepsilon^{\beta \alpha} \boldsymbol{a}_{\beta} \times \boldsymbol{u}_{, \alpha}-(\dot{J} / J) \boldsymbol{n},
$$

where $\varepsilon^{\beta \alpha}$ is the contravariant Levi-Civita permutation tensor $\left(\varepsilon^{\beta \alpha}=e^{\beta \alpha} / \sqrt{a}\right.$, with $e^{12}=-e^{21}=1$ and $\left.e^{11}=e^{22}=0\right)$; and

$$
\dot{W}=\dot{U}-\dot{\boldsymbol{e}}_{a}(\boldsymbol{r}) \cdot \boldsymbol{\pi},
$$

with

$$
\dot{\boldsymbol{e}}_{a}=\left(\operatorname{grad} \boldsymbol{e}_{a}\right)_{\mid \omega} \boldsymbol{u} \text { and } \dot{U}=U_{H} \dot{H}+U_{K} \dot{K},
$$

in which we have invoked $U_{\eta}=\mathbf{0}$ (cf. (44) and (46)1); and, from (49),

$$
U_{H}=2 k H \quad \text { and } \quad U_{K}=\bar{k} .
$$

Expressions for the variations $\dot{H}$ and $\dot{K}$ are known [Steigmann et al. 2003] and will be recalled in the next subsection. To facilitate their representation we use the decomposition

$$
\boldsymbol{u}=u^{\alpha} \boldsymbol{a}_{\alpha}+w \boldsymbol{n},
$$

where $u^{\alpha}$ and $w$ respectively are the tangential and normal variations of the position field.

4.1. Tangential variations. For tangential variations we have $w=0$ and

$$
\dot{J} / J=u_{; \alpha}^{\alpha}, \quad \dot{H}=u^{\alpha} H_{, \alpha} \quad \text { and } \quad \dot{K}=u^{\alpha} K_{, \alpha}
$$

[Steigmann et al. 2003]. Thus,

$$
(W+\lambda) \dot{J} / J=\left[(W+\lambda) u^{\alpha}\right]_{; \alpha}-u^{\alpha}(W+\lambda)_{, \alpha},
$$

where

$$
W_{, \alpha}=U_{, \alpha}-\boldsymbol{a}_{\alpha} \cdot\left(\operatorname{grad} \boldsymbol{e}_{a}\right)_{\mid \omega} \boldsymbol{\pi}-\boldsymbol{e}_{a} \cdot \boldsymbol{\pi}, \alpha ;
$$

whereas, with $\dot{\pi}=0$,

$$
\dot{W}=u^{\alpha}\left[U_{H} H_{, \alpha}+U_{K} K_{, \alpha}-\boldsymbol{a}_{\alpha} \cdot\left(\operatorname{grad} \boldsymbol{e}_{a}\right)_{\mid \omega} \boldsymbol{\pi}\right] .
$$


We thus reach

$$
\begin{aligned}
\dot{W}+(W+\lambda) \dot{J} / J & \\
& =\left[(W+\lambda) u^{\alpha}\right]_{; \alpha}+u^{\alpha}\left(U_{H} H_{, \alpha}+U_{K} K_{, \alpha}-U_{, \alpha}-\lambda_{, \alpha}+\boldsymbol{e}_{a} \cdot \boldsymbol{\pi}_{, \alpha}\right) .
\end{aligned}
$$

Here we use the fact that $U_{\eta}$ vanishes in equilibrium, together with (48) and the symmetry of $\mathbb{P}$, to derive

$$
U_{, \alpha}=U_{H} H_{, \alpha}+U_{K} K_{, \alpha}+\boldsymbol{e}_{a} \cdot \mathbb{P} \boldsymbol{\pi}_{, \alpha},
$$

which furnishes

$$
\dot{W}+(W+\lambda) \dot{J} / J=\left[(W+\lambda) u^{\alpha}\right]_{; \alpha}+u^{\alpha}\left[\left(\boldsymbol{e}_{a} \cdot \boldsymbol{n}\right)\left(\boldsymbol{n} \cdot \boldsymbol{\pi}_{, \alpha}\right)-\lambda_{, \alpha}\right] .
$$

To reduce the term in (50) involving $\varphi$, we use (53) to obtain [Steigmann 2013]

$$
\dot{\boldsymbol{n}}=\varepsilon^{\beta \alpha} b_{\lambda \alpha} u^{\lambda} \boldsymbol{a}_{\beta} \times \boldsymbol{n},
$$

where $\varepsilon_{\beta \lambda}$ is the covariant permutation tensor, together with $\varepsilon^{\beta \alpha} \varepsilon_{\beta \lambda}=\delta_{\lambda}^{\alpha}$ (the Kronecker delta). This and

$$
\boldsymbol{n} \times \boldsymbol{a}_{\beta}=\varepsilon_{\beta \gamma} \boldsymbol{a}^{\gamma}
$$

yield

$$
\dot{\boldsymbol{n}}=-b_{\lambda \alpha} u^{\lambda} \boldsymbol{a}^{\alpha}
$$

which combines with (47) to deliver

$$
\varphi \boldsymbol{\pi} \cdot \dot{\boldsymbol{n}}=-\left(\boldsymbol{n} \cdot \boldsymbol{e}_{a}\right) b_{\alpha \beta} \pi^{\beta} u^{\alpha} .
$$

On the other hand, the constraint (21) implies that

$$
\boldsymbol{\pi}_{, \alpha}=\pi_{; \alpha}^{\beta} \boldsymbol{a}_{\beta}+b_{\alpha \beta} \pi^{\beta} \boldsymbol{n},
$$

where $(\cdot)_{; \alpha}$ is the covariant derivative on $\omega$. Accordingly,

$$
\dot{W}+(W+\lambda) \dot{J} / J+\varphi \boldsymbol{\pi} \cdot \dot{\boldsymbol{n}}=\left[(W+\lambda) u^{\alpha}\right]_{; \alpha}-u^{\alpha} \lambda_{, \alpha} .
$$

Using Stokes' theorem, the surface integral over $\omega$ of the first term on the right-hand side may be represented as an integral over the edge $\partial \omega$. Remarkably, the Euler equations emerging from (42) under tangential variations are then given simply by $\lambda_{, \alpha}=0$; i.e.,

$$
\lambda \text { is constant on } \omega \text {, }
$$

as in the classical Canham-Helfrich theory for uniform lipid bilayers in the absence of electromagnetic effects [Steigmann et al. 2003; Dharmavaram and Healey 2015]. Edge conditions are discussed below. 
4.2. Normal variations. In this case $\boldsymbol{u}=\boldsymbol{w n}$, yielding [Steigmann et al. 2003]

$$
\begin{aligned}
\dot{J} / J & =-2 H w, \\
2 \dot{H} & =\Delta w+w\left(4 H^{2}-2 K\right), \\
\dot{K} & =2 K H w+\left(\tilde{b}^{\alpha \beta} w_{, \alpha}\right)_{; \beta},
\end{aligned}
$$

where, for any scalar field $\xi$,

$$
\Delta \xi=\frac{1}{\sqrt{a}}\left(\sqrt{a} a^{\alpha \beta} \xi_{, \beta}\right)_{, \alpha}
$$

is the surface Laplacian in which $a^{\alpha \beta}$ is the dual metric.

Recalling that $U_{\eta}$ vanishes at equilibrium and noting that $\pi$ is fixed in the present class of variations, after a lengthy calculation presented explicitly in [Steigmann et al. 2003] we reach

$$
\begin{aligned}
\dot{W}+(W+\lambda) \dot{J} / J=w[ & \Delta\left(\frac{1}{2} U_{H}\right)+\left(U_{K}\right)_{; \alpha \beta} \tilde{b}^{\alpha \beta}+U_{H}\left(2 H^{2}-K\right) \\
& \left.+2 H\left(K U_{K}-W\right)-2 H \lambda-\boldsymbol{n} \cdot\left(\operatorname{grad} \boldsymbol{e}_{a}\right)_{\mid \omega} \boldsymbol{\pi}\right] \\
+ & {\left[\left(\frac{1}{2} U_{H} a^{\alpha \beta}+U_{K} \tilde{b}^{\alpha \beta}\right) w_{, \alpha}\right]_{; \beta} } \\
& -\left\{\left[\left(U_{H}\right)_{, \beta} a^{\alpha \beta}+\left(U_{K}\right)_{, \beta} \tilde{b}^{\alpha \beta}\right] w\right\}_{; \alpha} .
\end{aligned}
$$

Here $(\cdot)_{; \alpha \beta}$ is the second covariant derivative on $\omega$ and

$$
\tilde{\boldsymbol{b}}=2 H \mathbf{1}-\boldsymbol{b}
$$

is the cofactor of the curvature tensor.

Further, (53) now gives [Steigmann 2013]

$$
\dot{\boldsymbol{n}}=-\boldsymbol{a}^{\alpha} w_{, \alpha},
$$

yielding

$$
\varphi \boldsymbol{\pi} \cdot \dot{\boldsymbol{n}}=w\left(\varphi \pi^{\alpha}\right)_{; \alpha}-\left(\varphi \pi^{\alpha} w\right)_{; \alpha} .
$$

Combining this with (41) and writing the integrals of the divergences as boundary integrals, from (42) the relevant Euler-Lagrange equation is found to be

$$
\begin{aligned}
\Delta\left(\frac{1}{2} U_{H}\right)+\left(U_{K}\right)_{; \alpha \beta} \tilde{b}^{\alpha \beta}+U_{H}\left(2 H^{2}-K\right) & \\
+2 H\left(\boldsymbol{\pi} \cdot \boldsymbol{e}_{a}+K U_{K}-U\right)-2 H \lambda & +\left(\varphi \pi^{\alpha}\right)_{; \alpha} \\
& =p+\boldsymbol{n} \cdot\left(\operatorname{grad} \boldsymbol{e}_{a}\right)_{\mid \omega} \boldsymbol{\pi}
\end{aligned}
$$

where

$$
\left(\varphi \pi^{\alpha}\right)_{; \alpha}=\frac{1}{\sqrt{a}}\left(\sqrt{a} \varphi \pi^{\alpha}\right)_{, \alpha} .
$$

This generalizes the well-known shape equation of the conventional theory [OuYang et al. 1999; Agrawal and Steigmann 2009]. For the particular energy given 
by (49) it reduces to

$$
\begin{aligned}
k\left[\Delta H+2 H\left(H^{2}-K\right)\right]-D|\boldsymbol{\pi}|^{2} H+2 H\left(\boldsymbol{\pi} \cdot \boldsymbol{e}_{a}-\lambda\right) & +\left(\varphi \pi^{\alpha}\right)_{; \alpha} \\
& =p+\boldsymbol{n} \cdot\left(\operatorname{grad} \boldsymbol{e}_{a}\right)_{\mid \omega} \boldsymbol{\pi},
\end{aligned}
$$

with $\varphi=\boldsymbol{n} \cdot \boldsymbol{e}_{a}$. This may be simplified by using (47) and (48) to reach

$$
D \pi \cdot \boldsymbol{e}_{a}=\mathbb{P} \boldsymbol{e}_{a} \cdot \boldsymbol{e}_{a}=\left|\mathbb{P} \boldsymbol{e}_{a}\right|^{2}=\left|\boldsymbol{e}_{a}\right|^{2}-\varphi^{2} .
$$

Then,

$$
2 H \pi \cdot \boldsymbol{e}_{a}-D|\boldsymbol{\pi}|^{2} H=D^{-1} H\left(\left|\boldsymbol{e}_{a}\right|^{2}-\varphi^{2}\right) .
$$

\section{Edge conditions}

With (71) and (78) satisfied the residual virtual-work statement (42) is

$$
\int_{\partial \omega} \chi d s=B_{t}+B_{n}
$$

with

$$
B_{t}=\int_{\partial \omega}(W+\lambda) u^{\alpha} v_{\alpha} d s
$$

and

$$
\begin{aligned}
B_{n}=\int_{\partial \omega}\left(\frac{1}{2} U_{H} a^{\alpha \beta}+U_{K} \tilde{b}^{\alpha \beta}\right) v_{\beta} w_{, \alpha} d s & \\
& -\int_{\partial \omega}\left[\frac{1}{2}\left(U_{H}\right)_{, \beta} a^{\alpha \beta}+\left(U_{K}\right)_{, \beta} \tilde{b}^{\alpha \beta}+\varphi \pi^{\alpha}\right] v_{\alpha} w d s .
\end{aligned}
$$

The part $B_{n}$ of the boundary working given above is exactly as in [Agrawal and Steigmann 2009]. The part $B_{t}$ may be reduced to a more convenient form by using $u^{\alpha}=\boldsymbol{u} \cdot \boldsymbol{a}^{\alpha}$ with $\boldsymbol{a}^{\alpha}=v^{\alpha} \boldsymbol{v}+\tau^{\alpha} \boldsymbol{\tau}$, where $\boldsymbol{\tau}=\boldsymbol{n} \times \boldsymbol{v}$ is the unit tangent to $\partial \omega$. This satisfies $\boldsymbol{\tau}=d \boldsymbol{r}\left(\theta^{\alpha}(s)\right) / d s=\tau^{\alpha} \boldsymbol{a}_{\alpha}$, where $\tau^{\alpha}=d \theta^{\alpha} / d s$. Thus,

$$
u^{\alpha}=v^{\alpha} \boldsymbol{u} \cdot \boldsymbol{v}+\tau^{\alpha} \boldsymbol{u} \cdot \boldsymbol{\tau},
$$

yielding

$$
B_{t}=\int_{\partial \omega}(W+\lambda) \boldsymbol{v} \cdot \boldsymbol{u} d s
$$

For smooth edges the foregoing may be combined with $B_{n}$ to reduce the edgepower density to the compact form [Agrawal and Steigmann 2009]

$$
\chi=f \cdot u-M \tau \cdot \omega,
$$

where $\omega$ is the variation of the surface orientation $(\dot{n}=\omega \times n)$,

$$
M=\frac{1}{2} U_{H}+\kappa_{\tau} U_{K}
$$


is the bending couple per unit length on $\partial \omega$ (divided by $t$ in accordance with the remarks following (42)),

$$
\boldsymbol{f}=F_{v} \boldsymbol{v}+F_{\tau} \boldsymbol{\tau}+F_{n} \boldsymbol{n}
$$

and is the edge traction (force per unit length, divided by $t$ ) on $\partial \omega$, with

$$
\begin{aligned}
& F_{v}=W+\lambda-\kappa_{v} M, \quad F_{\tau}=-\tau M \text { and } \\
& F_{n}=\left(\tau U_{K}\right)_{, s}-\left(\frac{1}{2} U_{H}\right)_{, v}-\left(U_{K}\right)_{, \beta} \tilde{b}^{\alpha \beta} v_{\alpha}-\varphi \pi^{\alpha} v_{\alpha} .
\end{aligned}
$$

Here $(\cdot)_{, v}=v^{\alpha}(\cdot)_{, \alpha}$ and $(\cdot)_{, s}=\tau^{\alpha}(\cdot)_{, \alpha}=d(\cdot) / d s$ are the normal and tangential derivatives on the boundary.

Further,

$$
\tau=b^{\alpha \beta} \tau_{\alpha} v_{\beta}
$$

is the twist of the surface $\omega$ on the $\boldsymbol{v}, \boldsymbol{\tau}$-axes, whereas

$$
\kappa_{v}=b^{\alpha \beta} v_{\alpha} v_{\beta} \quad \text { and } \quad \kappa_{\tau}=b^{\alpha \beta} \tau_{\alpha} \tau_{\beta},
$$

respectively, are the normal curvatures of $\omega$ in the directions of $\boldsymbol{v}$ and $\boldsymbol{\tau}$.

For the energy defined by (39) and (49) the bending moment and edge forces are

$$
M=k H+\bar{k} \tau
$$

and

$$
\begin{aligned}
& F_{\nu}=k H^{2}+\bar{k} K+\frac{1}{2} D|\boldsymbol{\pi}|^{2}-\boldsymbol{e}_{a} \cdot \boldsymbol{\pi}+\lambda-\kappa_{\nu} M, \\
& F_{\tau}=-\tau M \quad \text { and } \quad F_{n}=\bar{k} \tau_{, s}-k H_{, v}-\varphi \pi^{\alpha} \nu_{\alpha} .
\end{aligned}
$$

The force and moment are assigned on parts of the boundary that are complementary with respect to $\partial \omega$, respectively, to those parts where position $r$ and surface orientation $\boldsymbol{n}$ are assigned.

\section{Examples: axisymmetric states}

6.1. Uniform applied field. We assume the length scale for the spatial variation of the applied field to be much larger than that of the overall dimensions of the lipid membrane, so that the applied field in the vicinity of the membrane is sensibly constant; we take

$$
\boldsymbol{e}_{a}=E \boldsymbol{k},
$$

with $E$ constant and $\boldsymbol{k}$ a fixed unit vector.

We seek an axisymmetric solution in the class of closed surfaces of revolution with the axis of symmetry parallel to the applied field. This is parametrized 
by meridianal arclength $s$, measured from the north pole, and azimuthal angle $\theta \in[0,2 \pi)$. Thus,

$$
\boldsymbol{r}(s, \theta)=r(s) \boldsymbol{e}_{r}(\theta)+z(s) \boldsymbol{k},
$$

where $r(s)$ is the radius from the axis of symmetry, $z(s)$ is the elevation above a base plane and $\left\{\boldsymbol{e}_{r}, \boldsymbol{e}_{\theta}, \boldsymbol{k}\right\}$, with $\boldsymbol{e}_{\theta}=\boldsymbol{k} \times \boldsymbol{e}_{r}$, is the usual polar orthonormal basis. Meridians and parallels of latitude are the curves on which $\theta$ and $s$, respectively, are constant. Because $s$ measures arclength along meridians, we have

$$
\left(r^{\prime}\right)^{2}+\left(z^{\prime}\right)^{2}=1
$$

where $(\cdot)^{\prime}=d(\cdot) / d s$; therefore there is $\psi(s)$ such that

$$
r^{\prime}(s)=\cos \psi \quad \text { and } \quad z^{\prime}(s)=\sin \psi .
$$

Proceeding as in [Agrawal and Steigmann 2009] we have

$$
\boldsymbol{v}=-\cos \psi \boldsymbol{e}_{r}-\sin \psi \boldsymbol{k}, \quad \boldsymbol{\tau}=-\boldsymbol{e}_{\theta}, \quad \boldsymbol{n}=\cos \psi \boldsymbol{k}-\sin \psi \boldsymbol{e}_{r},
$$

and

$$
\kappa_{v}=\psi^{\prime}, \quad \kappa_{\tau}=r^{-1} \sin \psi, \quad \tau=0 .
$$

The sum of the normal curvatures is twice the mean curvature $H(s)$; hence the differential equation

$$
r \psi^{\prime}=2 r H-\sin \psi
$$

Their product yields the Gaussian curvature $K(s)$; thus,

$$
K=H^{2}-\left(H-r^{-1} \sin \psi\right)^{2} .
$$

Following the procedure outlined in Section 4.1 of [Agrawal and Steigmann 2009] and noting that the spatial gradient of $\boldsymbol{e}_{a}$ vanishes, with some labor we reduce the shape equation (80) to

$$
\begin{aligned}
L^{\prime}=r\left\{p / k+(2 \lambda / k) H-\left(E^{2} / D k\right)\left[H\left(1+\cos ^{2} \psi\right)-\psi^{\prime} \sin ^{2} \psi\right]\right. \\
\left.-2 H\left(H-r^{-1} \sin \psi\right)^{2}\right\},
\end{aligned}
$$

with

$$
H^{\prime}=r^{-1} L
$$

We omit the details of the straightforward but lengthy derivation. The system to be solved thus consists of (99), (102), (104) and (105), for the functions $r, z, \psi$, $H$ and $L$. To render the number of differential equations consistent with the total number of side conditions, we append (cf. (71))

$$
\lambda^{\prime}=0 .
$$


Consider the equilibrium of a subsurface $\tilde{\omega} \subset \omega$ containing the pole, bounded by a parallel of latitude defined by $s=\tilde{s}$, where $s$ measures meridianal arclength from the pole. The force balance (modulo the multiplicative factor $t^{-1}$ ) is

$$
\int_{\tilde{\omega}}\left[p \boldsymbol{n}+\left(\operatorname{grad} \boldsymbol{e}_{a}\right)_{\mid \omega} \boldsymbol{\pi}\right] d a+\int_{\partial \tilde{\omega}} \boldsymbol{f} d u+F \boldsymbol{k}=\mathbf{0},
$$

where $u=r(\tilde{s}) \theta$ measures arclength around the perimeter of the parallel, $f$ is the force per unit length exerted on $\tilde{\omega}$ by the part $\omega \backslash \tilde{\omega}$ of the membrane, and $F$ is a point load acting at the pole and directed along the symmetry axis. Because parallels of latitude are lines of curvature on the membrane, the twist $\tau$ vanishes on $\partial \tilde{\omega}$ and (cf. (90) and (101) 3 )

$$
\boldsymbol{f}=F_{\nu} \tilde{\boldsymbol{v}}+F_{n} \boldsymbol{n},
$$

where $\tilde{\boldsymbol{v}}$ is the exterior unit normal to $\tilde{\omega}$ (the opposite of $\boldsymbol{v}$ in $\left.(100)_{1}\right)$ and $F_{v}, F_{n}$ are defined in (91). Thus,

$$
\boldsymbol{f}=\left(F_{\nu} \cos \psi-F_{n} \sin \psi\right) \boldsymbol{e}_{r}+\left(F_{\nu} \sin \psi+F_{n} \cos \psi\right) \boldsymbol{k},
$$

and the periodicity of $\boldsymbol{e}_{r}(\theta)$ yields

$$
\int_{\partial \tilde{\omega}} \boldsymbol{f} d u=2 \pi r(\tilde{s})\left(F_{\nu} \sin \psi+F_{n} \cos \psi\right) \boldsymbol{k} .
$$

The presumed boundedness of the integrand of the first term in (107), together with the conditions

$$
r(0)=0, \quad \psi(0)=0
$$

at the pole, imply that

$$
F / 2 \pi+\lim _{\tilde{s} \rightarrow 0}\left(r F_{n}\right)=0 .
$$

According to (48), the polarization at the pole is proportional to the projection of the applied field onto the plane with unit normal $\boldsymbol{k}$. This vanishes by virtue of (96), and (112) reduces to

$$
F / 2 \pi+k \lim _{\tilde{s} \rightarrow 0}\left(r H^{\prime}\right)=0, \quad \text { yielding } \quad L(0)=-F / 2 \pi k .
$$

A similar condition applies at the opposite pole of the membrane.

To solve the equations it is convenient to convert the independent variable from the meridianal arclength $s$ to the surface area $a(s)$ of the sector $[0, s]$, defined by

$$
a(s)=2 \pi \int_{0}^{s} r(t) d t .
$$

We have $a^{\prime}(s)=2 \pi r(s)$, which is positive on the domain, implying that $a$ and 
$s$ are in one-to-one correspondence and hence that the former may replace the latter as the independent variable. The constraint on surface area is then enforced simply by integrating the equations over the domain $[0, A]$, where $A$ is the assigned membrane area. The conversion of the equations is discussed in detail in [Agrawal and Steigmann 2009] and thus not presented here. To treat the equations numerically we adopt an assigned length scale $R$, say, which we take to be the radius of a spherical reference vesicle. This is equilibrated in the absence of pressure and applied electric field, provided that the associated value of lambda vanishes. This radius and the modulus $k$ are the parameters used to nondimensionalize the equations. The electric field enters the resulting system via the combination

$$
\bar{E}=E^{2} R^{2} / D k
$$

which is positive if the flexoelectric effect is weak, and negative if it is strong.

Figure 1 depicts membrane shapes obtained using the MATLAB boundary value problem solver applied to the foregoing differential equations. The boundary conditions are: zero radius and angle $\psi$, and vanishing point load, at the north pole; and zero radius and elevation, with $\psi=-\pi$, at the south pole. The reaction force at the south pole is given a posteriori by an appropriate adjustment to (120), if desired, and we impose zero lateral pressure. The effect of flexoelectricity at a given field strength manifests itself as a vertical elongation or compression of the membrane along the field direction, corresponding respectively to weak or strong flexoelectricity.

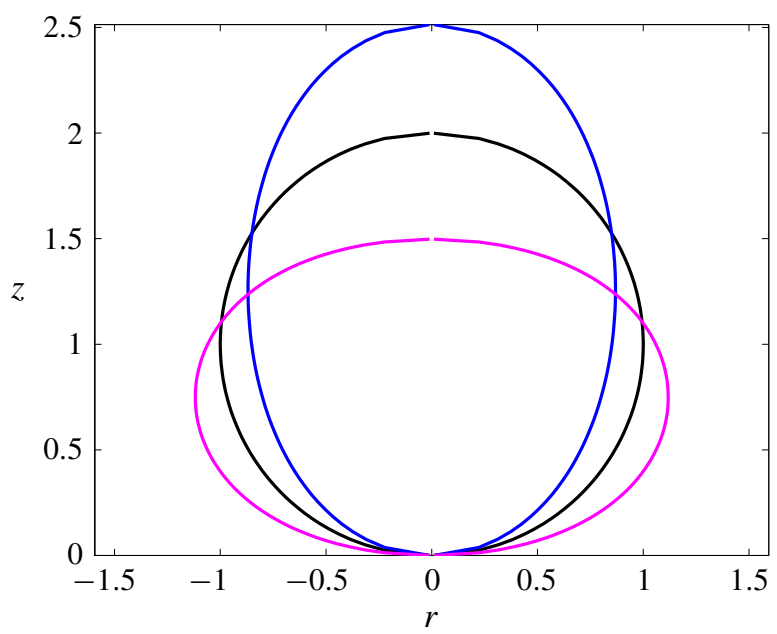

Figure 1. Vesicle subjected to uniform electric field. Black curve: original spherical vesicle. Blue curve: vesicle subjected to $\bar{E}=2.5$. Magenta solid curve: vesicle subjected to $\bar{E}=-2.5$. 
6.2. Remote point charge. We model the response of the membrane to a remote point charge located on the axis of symmetry, situated at the position

$$
\boldsymbol{y}_{c}=z_{c} \boldsymbol{k} \text {. }
$$

The associated electric potential and field are

$$
V_{a}(\boldsymbol{y})=\frac{E}{4 \pi \varepsilon_{0}\left|\boldsymbol{y}-\boldsymbol{y}_{c}\right|} \quad \text { and } \quad \boldsymbol{e}_{a}(\boldsymbol{y})=-\operatorname{grad} V_{a}=\frac{E}{4 \pi \varepsilon_{0}\left|\boldsymbol{y}-\boldsymbol{y}_{c}\right|^{2}} \boldsymbol{u}(\boldsymbol{y}) \text {, }
$$

respectively, where $E$ is the charge, $\varepsilon_{0}$ is the free-space permittivity, and

$$
\boldsymbol{u}(\boldsymbol{y})=\left(\boldsymbol{y}-\boldsymbol{y}_{c}\right) /\left|\boldsymbol{y}-\boldsymbol{y}_{c}\right| \text {. }
$$

The induced polarization is given by

$$
D \pi=\boldsymbol{e}_{a}-\varphi \boldsymbol{n},
$$

where

$$
\varphi=\boldsymbol{n} \cdot \boldsymbol{e}_{a}=\frac{E}{4 \pi \varepsilon_{0}}\left[\left(z-z_{c}\right) \cos \psi-r \sin \psi\right] /\left[r^{2}+\left(z-z_{c}\right)^{2}\right]^{3 / 2} .
$$

Thus,

$$
\boldsymbol{\pi}=\delta\left(\cos \psi \boldsymbol{e}_{r}+\sin \psi \boldsymbol{k}\right),
$$

with

$$
\delta=\frac{E}{4 \pi \varepsilon_{0} D}\left[r \cos \psi+\left(z-z_{c}\right) \sin \psi\right] /\left[r^{2}+\left(z-z_{c}\right)^{2}\right]^{3 / 2} .
$$

This is used in (80), in the combination

$$
\left(\varphi \pi^{\alpha}\right)_{; \alpha}=\frac{1}{r}(r \varphi \delta)^{\prime} .
$$

From $(116)_{2}$ we derive

$$
\frac{4 \pi \varepsilon_{0}}{E} \operatorname{grad} \boldsymbol{e}_{a \mid \omega}=\frac{1}{\left|\boldsymbol{r}-\boldsymbol{y}_{c}\right|^{3}} \boldsymbol{I}-\frac{3}{\left|\boldsymbol{r}-\boldsymbol{y}_{c}\right|^{5}}\left(\boldsymbol{r}-\boldsymbol{y}_{c}\right) \otimes\left(\boldsymbol{r}-\boldsymbol{y}_{c}\right),
$$

and with a bit of effort we then obtain the loading term

$$
\boldsymbol{n} \cdot\left(\operatorname{grad} \boldsymbol{e}_{a \mid \omega}\right) \boldsymbol{\pi}=\frac{-3 E}{4 \pi \varepsilon_{0}\left|\boldsymbol{r}-\boldsymbol{y}_{c}\right|^{5}}\left[\boldsymbol{n} \cdot\left(\boldsymbol{r}-\boldsymbol{y}_{c}\right)\right]\left[\boldsymbol{\pi} \cdot\left(\boldsymbol{r}-\boldsymbol{y}_{c}\right)\right],
$$

with

$$
\begin{aligned}
& \boldsymbol{n} \cdot\left(\boldsymbol{r}-\boldsymbol{y}_{c}\right)=\left(z-z_{c}\right) \cos \psi-r \sin \psi, \\
& \boldsymbol{\pi} \cdot\left(\boldsymbol{r}-\boldsymbol{y}_{c}\right)=\delta\left[r \cos \psi+\left(z-z_{c}\right) \sin \psi\right] .
\end{aligned}
$$

We substitute these results into the shape equation (80), obtaining

$$
\begin{aligned}
L^{\prime}=r\{[p+\boldsymbol{n} \cdot & \left.\left(\operatorname{grad} \boldsymbol{e}_{a}\right)_{\mid \omega} \boldsymbol{\pi}-\frac{1}{r}(r \varphi \delta)^{\prime}\right] / k \\
& \left.+(2 \lambda / k) H-D^{-1} H\left(\left|\boldsymbol{e}_{a}\right|^{2}-\varphi^{2}\right)-2 H\left(H-r^{-1} \sin \psi\right)^{2}\right\} .
\end{aligned}
$$

Further, the condition $(113)_{2}$, connecting $L$ to the point load at a pole, remains valid in the present circumstances. The equations are nondimensionalized as before. 
In this case the charge intensity enters the resulting system in the combination

$$
\bar{E}=\left(\frac{E}{4 \pi \varepsilon_{0}}\right)^{2} / D k,
$$

the sign of which depends on the strength of the flexoelectric effect, as before.

We present two examples. In the first the spherical vesicle of the previous example is subjected to a point charge located at the dimensionless position $\bar{z}_{c}=-0.75$ below the membrane. The boundary conditions are unchanged, the pressure vanishes, and we consider only the case of weak flexoelectricity $(\bar{E}>0)$. The point charge is seen to attract the membrane against the support reaction at the south pole. Figure 2 depicts the associated membrane equilibria.

The second example concerns a flat disc acted upon by a point charge at position $\bar{z}_{c}=-0.50$, again situated below the membrane. This is equilibrated at vanishing charge and pressure. The areal incompressibility constraint is still operative, but here, simply for the sake of illustration, we solve the problem on the dimensionless arclength interval $s / R \in[0,0.5]$, with $R$ as in the previous examples. Accordingly, as the membrane deforms, the model accommodates areal incompressibility implicitly via recruitment of lipids through the boundary, so that the computational domain does not correspond in this instance to a fixed set of lipids. The boundary conditions are: zero radius, angle and point load at the pole (i.e., at $s / R=0$ ); and zero elevation and angle at the remote edge. We impose zero pressure and take the Lagrange multiplier $\lambda$ to be zero everywhere. The latter condition renders (106) redundant, and gives rise to a state-dependent force $F_{v}$ and moment $M$ (cf. (94) and $\left.(95)_{1}\right)$ at the edge of the (nonmaterial) domain.

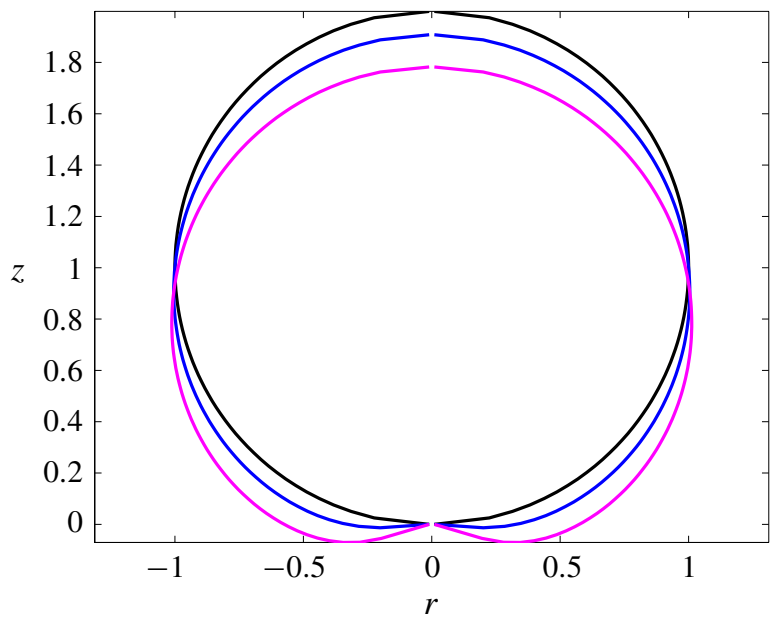

Figure 2. Effect of a point charge on the shape of a vesicle. Black curve: original spherical vesicle. Blue curve: vesicle subjected to $\bar{E}=1.75$. Magenta curve: vesicle subjected to $\bar{E}=2.08$. 


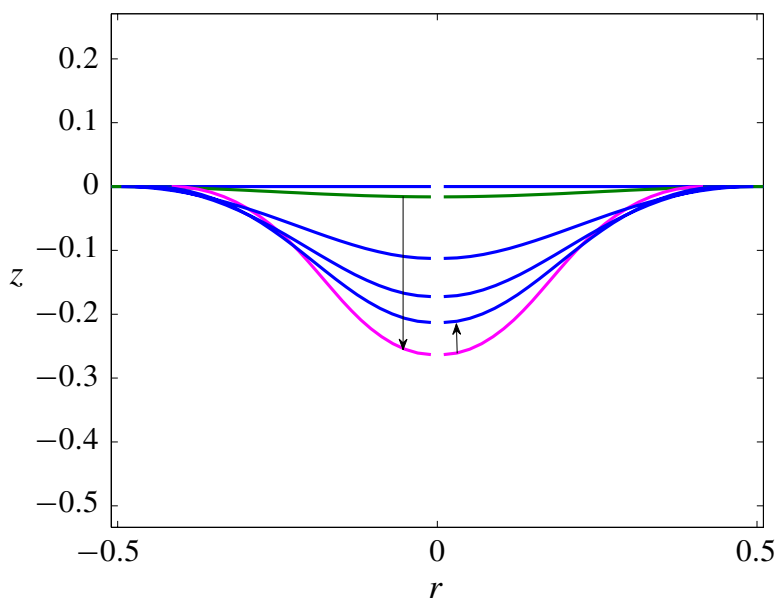

Figure 3. A flat patch of membrane with a nearby point charge. At $\bar{E}=0.5$ there is an instability. The patch goes from the green curve to the magenta curve. On increasing $\bar{E}$ further, the patch begins to flatten out. The maximum $\bar{E}$ used is 0.83 .

Figures 3 and 4 depict a sequence of predicted shapes arising in response to an increasing sequence of charge intensities in the case of weak flexoelectricity $(\bar{E}>0)$. At low values of the charge intensity the membrane is attracted to the charge, as in the previous example. We observe a sudden transition, at $\bar{E}=0.50$, from a slightly curved membrane to a strongly curved one. This trend, as indicated

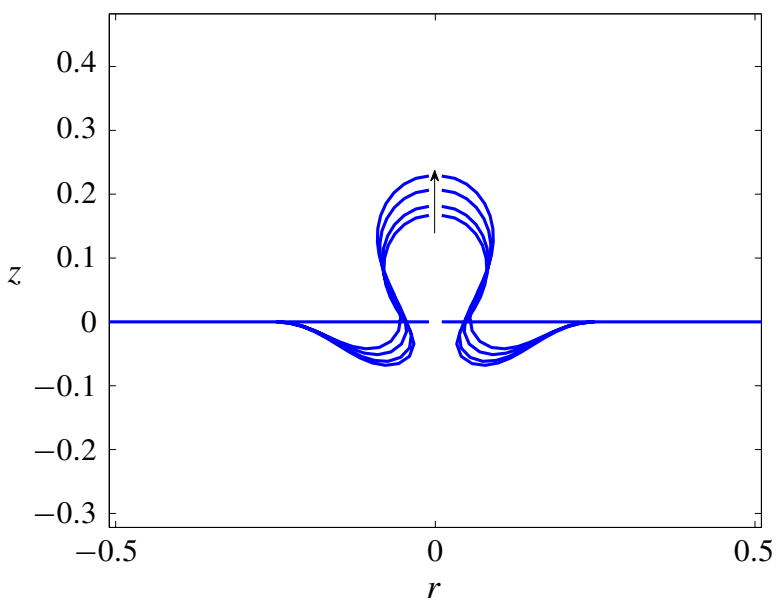

Figure 4. A flat patch of membrane with a nearby point charge. The arrow indicates the direction of increasing $\bar{E}$. The innermost shape corresponds to $\bar{E}=2.0$ and the outermost shape corresponds to $\bar{E}=2.5$. 
by the arrows in Figure 3, is reversed upon a further increase of the charge intensity, yielding ever flatter membranes, up to the value $\bar{E}=0.83$ (Figure 3 ).

We are unable to find equilibria for $\bar{E} \in(0.83,2.0)$ and thus offer the conjecture that no axisymmetric equilibria exist for charges in this interval. On further increase of the charge, however, we find the equilibria displayed in Figure 4, in which the arrow again indicates the trend under increasing charge intensity. These results support a conjecture to the effect that equilibria associated with the disjoint intervals of charge intensity are connected by a dynamic transition. On the second branch of equilibria, the membranes have formed a sequence of buds with ever-narrowing necks, situated, remarkably, above the initial disc shape, of a kind reminiscent of those observed in the process of endocytosis. This suggests that important biological processes such as endocytosis may be controlled, to some degree, by the action of suitable electric fields. We find the shapes displayed in these figures to be quite robust under shape perturbations in the setting of MATLAB, but we have not analyzed their stability. To study stability one should work with simulations carried out on a fixed material domain; i.e., on a patch of fixed area.

\section{Conclusions}

In this work, we formulate a generalized electromechanical theory of lipid membranes systematically from the three-dimensional liquid crystal theory. We derive the Euler-Lagrange equations and edge conditions required to solve boundary value problems in a coupled electromechanical setting. In contrast to earlier studies, we find that the lipid dipoles are primarily oriented in the tangent plane, as in the analogous magnetostatic setting. This has the effect of eliminating the self field from the leading-order two-dimensional model, and yields the important simplification that the self field can be computed a posteriori. Further, it provides justification for the widespread practice of suppressing the self field on an ad hoc basis. We also find that a spatially varying electric field does not lead to a spatial variation of the Lagrange multiplier associated with areal incompressibility. Numerical examples highlight the strong interplay between electric fields and membrane geometry.

\section{References}

[Agrawal and Steigmann 2008] A. Agrawal and D. J. Steigmann, "Coexistent fluid-phase equilibria in biomembranes with bending elasticity”, J. Elasticity 93:1 (2008), 63-80.

[Agrawal and Steigmann 2009] A. Agrawal and D. J. Steigmann, "Boundary-value problems in the theory of lipid membranes", Contin. Mech. Therm. 21:1 (2009), 57-82.

[Aihara and Miyazaki 1998] H. Aihara and J.-I. Miyazaki, "Gene transfer into muscle by electroporation in vivo", Nat. Biotechnol. 16:9 (1998), 867-870.

[Barham et al. 2012] M. Barham, D. J. Steigmann, and D. White, "Magnetoelasticity of highly deformable thin films: theory and simulation”, Int. J. Non-Linear Mech. 47:2 (2012), 185-196. 
[Brownell et al. 1985] W. E. Brownell, C. R. Bader, D. Bertrand, and Y. De Ribaupierre, "Evoked mechanical responses of isolated cochlear outer hair cells", Science 227:4683 (1985), 194-196.

[Bustamante et al. 2009] R. Bustamante, A. L. Dorfmann, and R. W. Ogden, "Nonlinear electroelastostatics: a variational framework", Z. Angew. Math. Phys. 60:1 (2009), 154-177.

[Cesana et al. 2015] P. Cesana, P. Plucinsky, and K. Bhattacharya, "Effective behavior of nematic elastomer membranes", Arch. Ration. Mech. Anal. 218:2 (2015), 863-905.

[Davalos et al. 2005] R. V. Davalos, L. M. Mir, and B. Rubinsky, "Tissue ablation with irreversible electroporation", Ann. Biomed. Eng. 33:2 (2005), 223-231.

[Dharmavaram and Healey 2015] S. Dharmavaram and T. J. Healey, "On the equivalence of local and global area-constraint formulations for lipid bilayer vesicles”, Z. Angew. Math. Phys. 66:5 (2015), 2843-2854.

[Dimova et al. 2007] R. Dimova, K. A. Riske, S. Aranda, N. Bezlyepkina, R. L. Knorr, and R. Lipowsky, "Giant vesicles in electric fields", Soft Matter 3:7 (2007), 817-827.

[Dimova et al. 2009] R. Dimova, N. Bezlyepkina, M. D. Jordo, R. L. Knorr, K. A. Riske, M. Staykova, P. M. Vlahovska, T. Yamamoto, P. Yang, and R. Lipowsky, "Vesicles in electric fields: some novel aspects of membrane behavior", Soft Matter 5:17 (2009), 3201-3212.

[Dorfmann and Ogden 2014] A. L. Dorfmann and R. W. Ogden, Nonlinear theory of electroelastic and magnetoelastic interactions, Springer, New York, NY, 2014.

[Ericksen 1961] J. L. Ericksen, "Conservation laws for liquid crystals", Trans. Soc. Rheol. 5 (1961), 23-34.

[Ericksen 1962] J. L. Ericksen, "Hydrostatic theory of liquid crystals", Arch. Ration. Mech. Anal. 9 (1962), 371-378.

[Ericksen 1976] J. L. Ericksen, "Equilibrium theory of liquid crystals", pp. 233-298 Advances in Liquid Crystals 2, Academic Press, New York, NY, 1976.

[Frischleder and Peinel 1982] H. Frischleder and G. Peinel, "Quantum-chemical and statistical calculations on phospholipids", Chem. Phys. Lipids 30:2-3 (1982), 121-158.

[Gao et al. 2008] L.-T. Gao, X.-Q. Feng, Y.-J. Yin, and H. Gao, "An electromechanical liquid crystal model of vesicles", J. Mech. Phys. Solids 56:9 (2008), 2844-2862.

[de Gennes and Prost 1992] P. G. de Gennes and J. Prost, The physics of liquid crystals, Oxford University Press, 1992.

[Gioia and James 1997] G. Gioia and R. D. James, "Micromagnetics of very thin films", Proc. R. Soc. Lond. A 453:1956 (1997), 213-223.

[Harland et al. 2015] B. Harland, W.-H. Lee, W. E. Brownell, S. X. Sun, and A. A. Spector, "The potential and electric field in the cochlear outer hair cell membrane", Med. Biol. Eng. Comput. 53:5 (2015), 405-413.

[Helfrich 1973] W. Helfrich, "Elastic properties of lipid bilayers: theory and possible experiments", Z. Naturforsch. 28 (1973), 693-703.

[Kim and Steigmann 2015] C.-I. Kim and D. J. Steigmann, "Distension-induced gradient capillarity in lipid membranes", Contin. Mech. Therm. 27:4-5 (2015), 609-621.

[Kovetz 2000] A. Kovetz, Electromagnetic theory, Oxford University Press, 2000.

[Kummrow and Helfrich 1991] M. Kummrow and W. Helfrich, "Deformation of giant lipid vesicles by electric fields", Phys. Rev. A 44:12 (1991), 8356-8360.

[Meyer 1969] R. B. Meyer, "Piezoelectric effects in liquid crystals", Phys. Rev. Lett. 22:18 (1969), 918-921.

[Mohammadi et al. 2014] P. Mohammadi, L. P. Liu, and P. Sharma, "A theory of flexoelectric membranes and effective properties of heterogeneous membranes", J. Appl. Mech. (ASME) 81:1 (2014), Article ID \#011007. 
[Naghdi 1972] P. M. Naghdi, "The theory of shells and plates", pp. 425-640 in Linear theories of elasticity and thermoelasticity, edited by C. Truesdell, Handbuch der Physik VIa/2, Springer, Berlin, 1972. Reprinted in 1984.

[Neumann et al. 1982] E. Neumann, M. Schaefer-Ridder, Y. Wang, and P. H. Hofschneider, "Gene transfer into mouse lyoma cells by electroporation in high electric fields", Euro. Mol. Biol. Organ. J. 1:7 (1982), 841-845.

[Ou-Yang et al. 1999] Z.-C. Ou-Yang, J.-X. Liu, and Y.-Z. Xie, Geometric methods in the elastic theory of membranes in liquid crystal phases, Advanced Series on Theoretical Physical Science 2, World Scientific, Singapore, 1999.

[Raphael et al. 2000] R. M. Raphael, A. S. Popel, and W. E. Brownell, "A membrane bending model of outer hair cell electromotility", Biophys. J. 78:6 (2000), 2844-2862.

[Rubinsky et al. 2007] B. Rubinsky, G. Onik, and P. Mikus, "Irreversible electroporation: a new ablation modality — clinical implications”, Technol. Cancer Res. Treat. 6:1 (2007), 37-48.

[Schmidt et al. 2012] D. Schmidt, J. del Mármol, and R. MacKinnon, "Mechanistic basis for low threshold mechanosensitivity in voltage-dependent $\mathrm{K}^{+}$channels", Proc. Nat. Acad. Sci. USA 109:26 (2012), 10352-10357.

[Seelig 1978] J. Seelig, “31 P nuclear magnetic resonance and the head group structure of phospholipids in membranes", Biochim. Biophys. Acta Biomembr. 515 (1978), 105-140.

[Sokolnikoff 1964] I. S. Sokolnikoff, Tensor analysis: theory and applications to geometry and mechanics of continua, Wiley, New York, NY, 1964.

[Steigmann 2013] D. J. Steigmann, "A model for lipid membranes with tilt and distension based on three-dimensional liquid crystal theory”, Int. J. Non-Linear Mech. 56 (2013), 61-70.

[Steigmann et al. 2003] D. J. Steigmann, E. Baesu, R. E. Rudd, J. Belak, and M. McElfresh, "On the variational theory of cell-membrane equilibria", Interfaces Free Bound. 5:4 (2003), 357-366.

[Toupin 1956] R. A. Toupin, "The elastic dielectric", J. Rational Mech. Anal. 5:6 (1956), 849-915.

[Truesdell and Toupin 1960] C. Truesdell and R. A. Toupin, "The classical field theories”, pp. 226793 in Principles of classical mechanics and field theory, edited by S. Flügge, Handbuch der Physik III/1, Springer, Berlin, 1960.

[Virga 1994] E. G. Virga, Variational theories for liquid crystals, Applied Mathematics and Mathematical Computation 8, Chapman \& Hall, London, 1994.

[Vlahovska 2010] P. M. Vlahovska, "Nonequilibrium dynamics of lipid membranes: deformation and stability in electric fields", pp. 101-146 Advances in Planar Lipid Bilayers and Liposomes 12, Academic Press, Oxford, 2010.

[Warshaviak et al. 2011] D. T. Warshaviak, M. J. Muellner, and M. Chachisvilis, "Effect of membrane tension on the electric field and dipole potential of lipid bilayer membrane", Biochim. Biophys. Acta Biomembr. 1808:10 (2011), 2608-2617.

[Weaver 2000] J. C. Weaver, "Electroporation of cells and tissues", IEEE Trans. Plasma Sci. 28:1 (2000), 24-33.

[Winterhalter and Helfrich 1988] M. Winterhalter and W. Helfrich, "Deformation of spherical vesicles by electric fields”, J. Colloid Interface Sci. 122:2 (1988), 583-586.

Received 19 Jun 2015. Revised 13 Nov 2015. Accepted 14 Dec 2015.

DAVID J. STEIGMANN: dsteigmann@berkeley.edu

Department of Mechanical Engineering, University of California, 6189 Etcheverry Hall No. 1740, Berkeley, CA 94720-1740, United States

Ashutosh AgRAwal: ashutosh@uh.edu

Department of Mechanical Engineering, University of Houston, Houston, TX 77204, United States
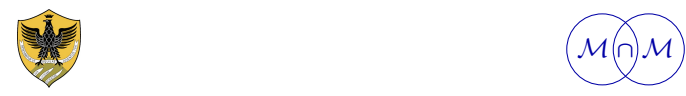
EDITORIAL BOARD

ANTONIO CARCATERRA

ERIC A. CARLEN

FRANCESCO DELL'ISOLA

RAFFAELE ESPOSITO

ALBERT FANNJIANG

Gilles A. FranCFORT

Pierangelo MARCATI

JEAN-JACQUES MARIGO

PETER A. MARKOWICH

MARTIN OSTOJA-STARZEWSKI

PIERRE SEPPECHER

DAVID J. STEIGMANN

PAUl STEINMANN

PierRe M. SuQueT

MANAGING EDITORS

MICOL AMAR

CORRADO LATTANZIO

ANGELA MADEO

MARTIN OSTOJA-STARZEWSKI

ADVISORY BOARD

ADNAN AKAY

Holm AltenBaCH

MICOL AMAR

HARM ASKES

TEODOR ATANACKOVIĆ

VICTOR BERDICHEVSKY

GUY BOUCHITTÉ

ANDREA BRAIDES

ROBERTO CAMASSA

MAURO CARFORE

ERIC DARVE

FELIX DARVE

ANNA DE MASI

GianPiEtro DEL Piero

EMMANUELE Di BENEDETTO

BERNOLD FIEDLER

IRENE M. GAMBA

DAVID Y. GAO

SERGEY GAVRILYUK

TIMOTHY J. HEALEY

DOMINIQUE JEULIN

ROGER E. KHAYAT

CORRADO LATTANZIO

ROBERT P. LIPTON

ANGELO LUONGO

ANGELA MADEO

JUAN J. MANFREDI

CARLO MARCHIORO

GÉRARD A. MAUGIN

ROBERTO NATALINI PATRIZIO NEFF

ANDREY PIATNITSKI

ERRICO PRESUTTI

MARIO PULVIRENTI

LUCIO RUSSO

Miguel A. F. SANJUAN

PATRICK SElVADURAI

ALEXANDER P. SEYRANIAN

MIROSLAV ŠILHAVÝ

GUIDO SWEERS

ANTOINETTE TORDESILLAS

LEV TRUSKINOVSKY

JUAN J. L. VELÁZQUEZ VINCENZO VESPRI ANGELO VULPIANI msp.org/memocs

Università di Roma "La Sapienza", Italia

Rutgers University, USA

(CO-CHAIR) Università di Roma "La Sapienza", Italia

(TREASURER) Università dell'Aquila, Italia

University of California at Davis, USA

(CO-CHAIR) Université Paris-Nord, France

Università dell'Aquila, Italy

École Polytechnique, France

DAMTP Cambridge, UK, and University of Vienna, Austria

(CHAIR MANAGING EDITOR) Univ. of Illinois at Urbana-Champaign, USA

Université du Sud Toulon-Var, France

University of California at Berkeley, USA

Universität Erlangen-Nürnberg, Germany

LMA CNRS Marseille, France

Università di Roma "La Sapienza", Italia

Università dell'Aquila, Italy

Université de Lyon-INSA (Institut National des Sciences Appliquées), France

(CHAIR MANAGING EDITOR) Univ. of Illinois at Urbana-Champaign, USA

Carnegie Mellon University, USA, and Bilkent University, Turkey

Otto-von-Guericke-Universität Magdeburg, Germany

Università di Roma "La Sapienza", Italia

University of Sheffield, UK

University of Novi Sad, Serbia

Wayne State University, USA

Université du Sud Toulon-Var, France

Università di Roma Tor Vergata, Italia

University of North Carolina at Chapel Hill, USA

Università di Pavia, Italia

Stanford University, USA

Institut Polytechnique de Grenoble, France

Università dell'Aquila, Italia

Università di Ferrara and International Research Center MEMOCS, Italia

Vanderbilt University, USA

Freie Universität Berlin, Germany

University of Texas at Austin, USA

Federation University and Australian National University, Australia

Université Aix-Marseille, France

Cornell University, USA

École des Mines, France

University of Western Ontario, Canada

Università dell' Aquila, Italy

Louisiana State University, USA

Università dell'Aquila, Italia

Université de Lyon-INSA (Institut National des Sciences Appliquées), France University of Pittsburgh, USA

Università di Roma "La Sapienza”, Italia

Université Paris VI, France

Istituto per le Applicazioni del Calcolo "M. Picone", Italy

Universität Duisburg-Essen, Germany

Narvik University College, Norway, Russia

Università di Roma Tor Vergata, Italy

Università di Roma "La Sapienza”, Italia

Università di Roma “Tor Vergata”, Italia

Universidad Rey Juan Carlos, Madrid, Spain

McGill University, Canada

Moscow State Lomonosov University, Russia

Academy of Sciences of the Czech Republic

Universität zu Köln, Germany

University of Melbourne, Australia

École Polytechnique, France

Bonn University, Germany

Università di Firenze, Italia

Università di Roma La Sapienza, Italia

MEMOCS (ISSN 2325-3444 electronic, 2326-7186 printed) is a journal of the International Research Center for the Mathematics and Mechanics of Complex Systems at the Università dell'Aquila, Italy.

Cover image: "Tangle” by $\odot$ John Horigan; produced using the Context Free program (contextfreeart.org).

PUBLISHED BY

7 mathematical sciences publishers

nonprofit scientific publishing

http://msp.org/

(C) 2016 Mathematical Sciences Publishers 
Mathematics and Mechanics of Complex Systems vol. 4 no. 1

Gradient materials with internal constraints

Albrecht Bertram and Rainer Glüge

Unified geometric formulation of material uniformity and evolution

Marcelo Epstein and Manuel de León

Electromechanics of polarized lipid bilayers

David J. Steigmann and Ashutosh Agrawal

Orthogonal polynomials and Riesz bases applied to the solution of Love's equation

Pierluigi Vellucci and Alberto Maria Bersani

Modeling capillary hysteresis in unsatured porous media

Gérard Gagneux and Olivier Millet

Discrete double-porosity models for spin systems

Andrea Braides, Valeria Chiadò Piat and Margherita Solci

Correction to "On the theory of diffusion and swelling in finitely deforming elastomers"

Gary J. Templet and David J. Steigmann

MEMOCS is a journal of the International Research Center for the Mathematics and Mechanics of Complex Systems at the Università dell' Aquila, Italy.

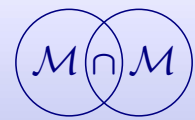

